\title{
Islet transplantation ameliorates diabetes-induced testicular interstitial fibrosis and is associated with inhibition of TGF-ß1/Smad2 pathway in a rat model of type 1 diabetes
}

\author{
YUAN-CAI ZHENG ${ }^{1}$, YI-LING FENG ${ }^{2}$, YI-HONG WANG ${ }^{2}$, LI-JUN KONG ${ }^{3}$, MING-SHI ZHOU $^{3}$, \\ MIN-MIN WU ${ }^{3}$, CHENG-YANG LIU ${ }^{4}$, HUA-CHUN WENG ${ }^{2,5}$ and HONG-WEI WANG ${ }^{3}$
}

Departments of ${ }^{1}$ Urology and ${ }^{2}$ Pediatrics, ${ }^{3}$ Key Laboratory of Diagnosis and Treatment of Severe Hepato-Pancreatic Diseases of Zhejiang Province, The First Affiliated Hospital of Wenzhou Medical University, Wenzhou, Zhejiang 325000, P.R. China; ${ }^{4}$ Department of Surgery, Perelman School of Medicine at the University of Pennsylvania, Philadelphia, PA 19104-5160, USA; ${ }^{5}$ The College of Medical Technology, Shanghai University of Medicine and Health Sciences, Shanghai 200000, P.R. China

Received August 16, 2020; Accepted February 3, 2021

DOI: $10.3892 / \mathrm{mmr} .2021 .12015$

\begin{abstract}
Islet transplantation (IT) is considered the most effective endocrine replacement therapy for diabetes mellitus (DM). Studies have demonstrated that IT can repair testicular structural injury caused by inflammatory and oxidative stress in a diabetic rat model. However, highly effective exogenous antioxidant and anti-inflammatory drugs can achieve this effect. Testicular interstitial fibrosis caused by long-term hyperglycemia is however difficult to reverse or recover. Thus far, there are no effective drugs that prevent or relieve testicular interstitial fibrosis. Therefore, it is necessary to explore the potential benefit of IT on testicular interstitial fibrosis induced by $\mathrm{DM}$ and its underlying molecular mechanisms. In the present study, Wistar rats were used to establish a DM model by intraperitoneal injection of streptozotocin. The diabetic models then underwent IT or received insulin treatment after 12 weeks. IT was more effective than insulin treatment in ameliorating diabetic-induced testicular interstitial fibrosis, Leydig cells apoptosis, testosterone deficiency and poor sperm motility. IT and insulin treatment both significantly inhibited the upregulation of TGF- $\beta 1$ and phosphorylated Smad 2 in DM, with IT being more effective than insulin. The present
\end{abstract}

Correspondence to: Dr Hong-Wei Wang, Key Laboratory of Diagnosis and Treatment of Severe Hepato-Pancreatic Diseases of Zhejiang Province, The First Affiliated Hospital of Wenzhou Medical University, 322 Nanbaixiang Street, Wenzhou, Zhejiang 325000, P.R. China

E-mail: 15258085168@163.com

Professor Hua-Chun Weng, Department of Pediatrics, The First Affiliated Hospital of Wenzhou Medical University, 322 Nanbaixiang Street, Wenzhou, Zhejiang 325000, P.R. China

E-mail:wenghc@sumhs.edu.cn

Key words: diabetes mellitus, islet transplantation, testicular dysfunction, interstitial fibrosis, TGF- $\beta 1 /$ Smad2 signaling study's findings proved that IT effectively protects diabeticinduced testicular interstitial fibrosis probably by inhibiting the TGF- $\beta 1 / \mathrm{Smad} 2$ signaling pathway, which offers hope in male patients with DM complicating with testicular interstitial fibrosis.

\section{Introduction}

Diabetes mellitus (DM) is a chronic metabolic disease characterized by prolonged hyperglycemia due to impaired insulin secretion or loss of insulin-producing islet $\beta$ cells (1). Investigation of DM in humans and animal models demonstrates that long-term hyperglycemia induces nephropathy, retinopathy, neuropathy and angiopathy (2). Recent studies demonstrate that DM also causes male infertility through testicular cell apoptosis, downregulation of testosterone level and reduction of libido $(3,4)$. Diabetic testicular dysfunction is one of the most common complications in male patients with DM, $90 \%$ having varying degrees of reproductive dysfunction (5). With an increase in DM cases and the general delay in childbearing, the fertility problems caused by diabetic testicular dysfunction are increasing (6). Therefore, it is important to explore the molecular mechanisms of diabetic testicular dysfunction and to develop effective treatment strategies to improve the fertility and quality of life in male patients with DM.

Studies have demonstrated that oxidative stress and inflammation are the main causes of testicular dysfunction in DM $(7,8)$. However, few studies have paid attention to testicular interstitial fibrosis in DM. Testicular interstitial fibrosis destroys the spermatogenic environment of the testis, which impairs testosterone secretion and spermatogenesis resulting in male infertility and sexual dysfunction $(9,10)$. The oxidative stress and inflammatory reactions in testicular tissues induced by DM can be inhibited by highly effective exogenous antioxidants and anti-inflammatory drugs $(11,12)$. However, testicular interstitial fibrosis caused by long-term high glucose levels is difficult to repair $(9,10)$. Testicular interstitial fibrosis is a necessary process for the development of diabetic testicular 
dysfunction characterized by irreversible oligozoospermia and persistent poor sperm motility $(10,13)$. There are no reported strategies for the prevention or treatment of diabetic testicular interstitial fibrosis. Hence, researchers have a difficult task in developing reliable methods for treating diabetic testicular interstitial fibrosis.

Islet transplantation (IT) is currently the most effective method for clinical treatment of various chronic complications of DM (14). Previous studies have demonstrated that IT can ameliorate and even reverse diabetic complications, including nephropathy, retinopathy and neuropathy in the early stages (15-17). Research has also revealed that IT improves testicular injury in diabetic rats through antioxidant stress and anti-inflammatory effects (18). However, restoration or reversal of DM-induced testicular interstitial fibrosis by IT remains to be elucidated.

TGF- $\beta 1$ is a ubiquitous cytokine that regulates cell growth and evidence suggests that TGF- $\beta 1$ is implicated in reproductive dysfunction through the activation of testicular fibroblasts and induction of sperm apoptosis $(19,20)$. The activation of TGF- $\beta 1$ signal transduction mainly depends on the phosphorylation of the Smad protein (21). TGF- $\beta 1$ activates type I receptor-phosphorylated (p-) Smad2, which interacts with Smad3 and Smad4 and then translocate to the nucleus for active transcription of fibrotic related genes, such as Collagen Type I $\alpha 1$ Chain (COL1A1), Collagen Type III $\alpha 1$ Chain (COL3A1), connective tissue growth factor (CTGF) and fibronectin $(22,23)$. In addition, CTGF and $\alpha$-smooth muscle actin $(\alpha-S M A)$ are important characteristics in fibrosis formation. CTGF, which is upregulated by activation of TGF- $\beta 1$, is a vital mediator in fibroblasts activation including differentiation, proliferation, adhesion and extracellular matrix (ECM) synthesis (24). High expression of $\alpha$-SMA is present in fibroblasts activation and promotes the deposition of Collal and Col3a1, leading to interstitial fibrosis $(25,26)$.

The present study investigated the reversal of testicular interstitial fibrosis in rats treated with IT at an advanced diabetic stage and the underlying mechanisms. It was demonstrated that IT could restore testicular interstitial fibrosis, Leydig cells apoptosis, testosterone deficiency and sperm motility in the rat model of type 1 diabetes, which had a close association with the recovery of testicular structure and function injury. The present study also discussed that the impact of IT in the testis of diabetic rats by reducing diabetic-induced testicular interstitial fibrosis and Leydig cells apoptosis may be through inhibiting the TGF- $\beta 1 / \mathrm{Smad} 2$ signaling pathway.

\section{Materials and methods}

Animals. A total of 42 healthy, clean grade, 8-week-old male Wistar rats weighing 200-220 g were purchased from the Experimental Animal Center of Wenzhou Medical University. All rats were housed with a $12 \mathrm{~h}$ light/dark cycle at $24 \pm 1^{\circ} \mathrm{C}$ with $50-60 \%$ humidity and fed ad libitum for 1 week before the study began. All animal experiments were performed according to the regulations of the Animal Experimental Ethical Inspection of Laboratory Animal Centre of Wenzhou Medical University (ID no. wydw-2017-0008) and were performed following the 'Guide for the Care and Use of Laboratory Animals' (27).
Diabetic models and groups. DM was induced by a single intraperitoneal injection of streptozotocin (STZ; Sigma-Aldrich; Merck KGaA; $50 \mathrm{mg} / \mathrm{kg}$ body weight) in sodium citrate buffer $(\mathrm{pH}=4.5)$. After 3 days, tail vein blood was collected for the detection of plasma blood glucose levels using an Accu-Check Active glucometer (Roche Diagnostics). Successful establishment of experimental diabetic rat models was identified as a non-fasted blood glucose concentration $\geq 16.67 \mathrm{mmol} / 1$ recorded for 3 consecutive days $(17,20)$. Then, 12 weeks after the diabetic models were established, the rats were divided into four groups. The first group comprised normal control (NC) rats $(n=6)$. The second group comprised DM rats $(n=6)$. The third group comprised INS rats $(n=6)$ that were treated with insulin (WanBang Biopharmaceuticals, Co., Ltd.) at a dose of $3 \mathrm{U}$ per injection given at 9 a.m. and 9 p.m. every day. In the fourth group $(n=6)$, IT was performed. The remaining 18 rats were used as IT donors and three donor rats matched one recipient rat. Sham operations were also performed at the same time IT was performed in the NC, untreated DM and the INS groups. After 4 weeks, all rats were anesthetized with isoflurane (3.5\% for induction and $2.5 \%$ for maintenance). Afterwards, large amounts of arterial blood was quickly taken from the abdominal aorta of rats $(\sim 5-7 \mathrm{ml}$ per rat) and then rats were sacrificed in the form of an immediate removal of the heart and arterial blood and testicular tissues were collected for detection.

Islet transplantation. IT was performed using a previously described procedure (28). Briefly, the donor rats were anesthetized with isoflurane $3.5 \%$ for induction and $2.5 \%$ for maintenance) and then sacrificed with the heart removed immediately after their arterial blood was rapidly taken from the abdominal aorta ( $\sim 5-7 \mathrm{ml}$ for each rat). Afterwards, the pancreas was exposed and injected with $8 \mathrm{ml}$ collagenase $\mathrm{V}$ (Sigma-Aldrich; Merck KGaA; 0.8 mg/ml, dissolved in Hank's solution) through the common bile duct. The pancreas was then separated from the surrounding tissues and digested with $2 \mathrm{ml}$ collagenase $\mathrm{V}$ at $37^{\circ} \mathrm{C}$. The islets were then washed, purified and centrifuged for $5 \mathrm{~min}$ with the speed of $200 \mathrm{x} \mathrm{g}$ at room temperature and transferred to a black glass culture dish for manual selection. The final purified islets were cultured in RPMI-1640 (Gibco; Thermo Fisher Scientific, Inc.) containing $10 \%$ FBS, 2 mM L-glutamate and $100 \mathrm{U} / \mathrm{ml}$ penicillin and streptomycin. Fluorescein diacetate-propidium iodide (FDAPI) staining (Sigma-Aldrich; Merck KGaA) was used to evaluate the activity of the purified islets, which were stained for $5 \mathrm{~min}$ at room temperature and then observed under a fluorescence microscope (Nikon ECLIPSE; Nikon Corporation; original magnification $\mathrm{x} 200)$. Prior transplantation, the recipient rats were anesthetized with isoflurane (3.5\% for induction and $2.5 \%$ for maintenance). Then, the kidney of the recipient rats was also exposed and the islets were transferred slowly and carefully through the kidney capsule. The incision was then sutured layer-by-layer. Subsequently, 4 weeks after IT, immunochemistry and hematoxylin and eosin (H\&E) staining were used to assess insulin secretion.

Detection of sperm count and sperm motility. The right epididymis of the rats was obtained and placed in a culture dish. The epididymis was obtained by gently cutting from the 
A

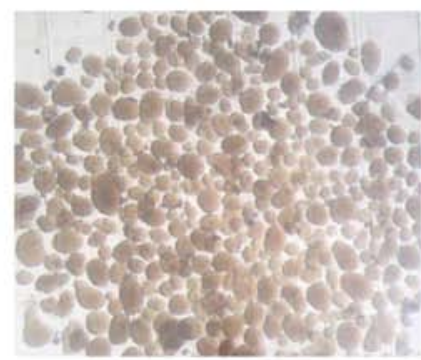

$(\times 40)$

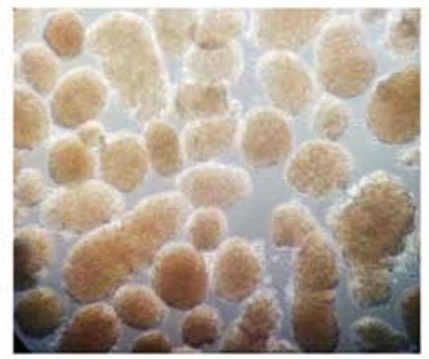

$(\times 400)$
B

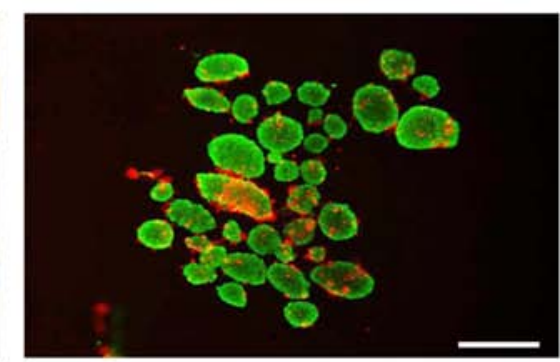

C

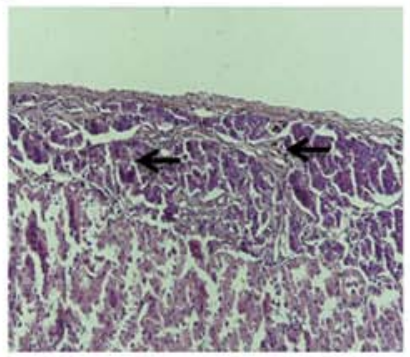

$(\times 200)$

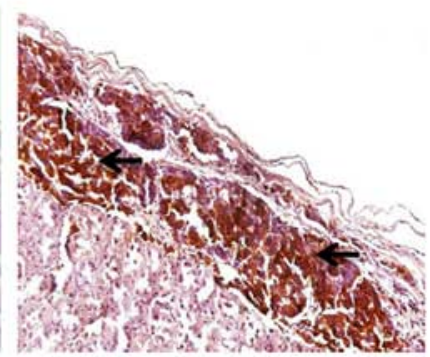

$(\times 200)$

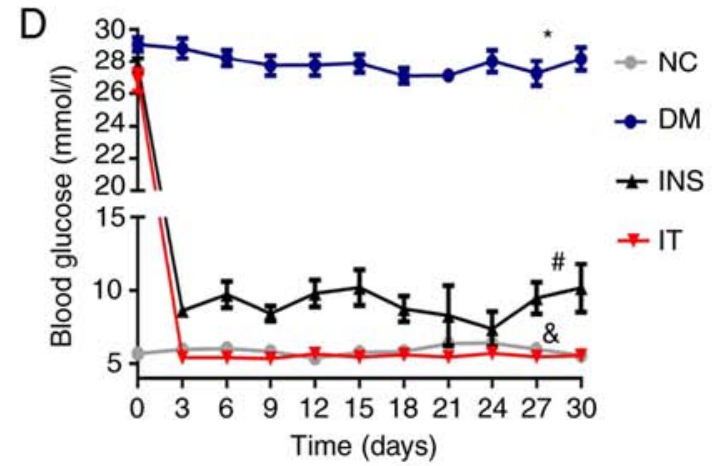

Figure 1. Morphology, purity and activities of isolated islets and changes in blood glucose levels in the different groups. (A) Morphology and purity of isolated islets. (B) The activity of isolated islets was determined through FDA-PI staining. Strong green fluorescence intensity indicates high activities of the isolated islets $(>95 \%)$. Scale bar $=25 \mu \mathrm{m}$. (C) Representative hematoxylin and eosin staining and immunohistochemical staining images reflect the stable colonization of islet grafts under the renal capsule and considerable release of insulin following transplantation respectively. (D) Blood glucose levels in diabetic rats remained high after 12 weeks. INS lowered blood glucose level with fluctuations. IT restored blood glucose levels to a near normal state, with negligible blood glucose fluctuations. " $\mathrm{P}<0.05$ vs. NC; ${ }^{~} \mathrm{P}<0.05$ vs. DM; ${ }^{\&} \mathrm{P}<0.05$ vs. INS. NC, normal control; DM, diabetes mellitus; INS, insulin treatment; IT, islet transplantation.

tail with ophthalmic scissors and was then diluted with $3 \mathrm{ml}$ of $37^{\circ} \mathrm{C}$ fertilization medium (cat. no. ART-1021; Sage In Vitro Fertilization, Inc.; CooperSurgical Company). The culture dish containing the epididymis was placed in an incubator with a constant temperature of $37^{\circ} \mathrm{C}$ for 10 min to enable sperm diffusion to obtain a suspension. Sperm suspension $(50 \mu \mathrm{l})$ was taken and diluted with $3 \mathrm{ml}$ of fertilization medium at $37^{\circ} \mathrm{C}$. Then, $10 \mu \mathrm{l}$ of the diluted suspension was put on a counting board and sperm count and motility analysis were performed under a light microscope (DM750; Leica Microsystems, Inc.; original magnification $\mathrm{x} 400)$.

Western blot analysis. Western blotting was performed as previously described (29). Briefly, proteins were extracted from the testicular tissue using RIPA buffer (Beyotime Institute of Biotechnology) complemented with $10 \%$ protease inhibitor cocktail (Sigma-Aldrich; Merck KGaA) and quantified by BCA protein assay (Beyotime Institute of Biotechnology). A total of $60 \mu \mathrm{g}$ proteins were separated by 10 or $12 \%$ sodium dodecyl sulfate-polyacrylamide gel electrophoresis and transferred to polyvinylidene difluoride membranes $(0.45 \mu \mathrm{l})$ for $90 \mathrm{~min}$ at $300 \mathrm{~mA}$ and then blocked with 5\% non-fat milk for $2 \mathrm{~h}$ at room temperature. After washing with Tris-buffered saline and $0.1 \%$ Tween 20 (TBS-T), the membrane was incubated with the following primary antibodies overnight: TGF- $\beta 1$ (1:1,000; Abcam; ab215715; Rabbit monoclonal), p-Smad2 (1:1,000; Abcam; ab184557; Rabbit monoclonal), Smad2
(1:1,000; Abcam; ab40855; Rabbit monoclonal), connective tissue growth factor (CTGF; 1:1,000; Abcam; ab227180; Rabbit polyclonal) and $\alpha$-SMA (1:1,000; Abcam; ab5694; Rabbit polyclonal). After washing with TBS-T, the membrane was then incubated with a horseradish peroxidase-conjugated secondary antibody (1:2,000; Santa Cruz Biotechnology, Inc.; cat. no. sc-2004; Goat anti-rabbit IgG-HRP) for $2 \mathrm{~h}$ at room temperature. Finally, the bands were visualized using enhanced chemiluminescence (Bio-Rad Laboratories, Inc.; cat. no. 1705040) and quantified with Image-Pro Plus 6.0 software (Media Cybernetics, Inc.).

Measurement of serum testosterone, luteinizing hormone ( $L H)$ and follicle-stimulating hormone (FSH) levels. Blood samples were collected following sacrifice of rats and centrifuged with the speed of 2,000 $\mathrm{xg}$ for $8 \mathrm{~min}$ at $4^{\circ} \mathrm{C}$ and the serum separated. Testosterone, $\mathrm{LH}$ and FSH levels in serum were assessed using ELISA kits (Shanghai Puji Biotechnology Co., Ltd.; Testosterone, cat. no. BP-E30610; LH, cat. no. BP-E920995; FSH, cat. no. BP-E30597) according to the manufacturers' protocols.

TUNEL analysis. TUNEL assay was utilized to detect apoptosis in the testes tissues according to the instructions of the TUNEL kit (Roche Applied Science). Briefly, testis sections $(5 \mu \mathrm{m})$ were rehydrated in a $100-70 \%$ ethanol gradient after dewaxing in xylene for $30 \mathrm{~min}$ and then incubated in 

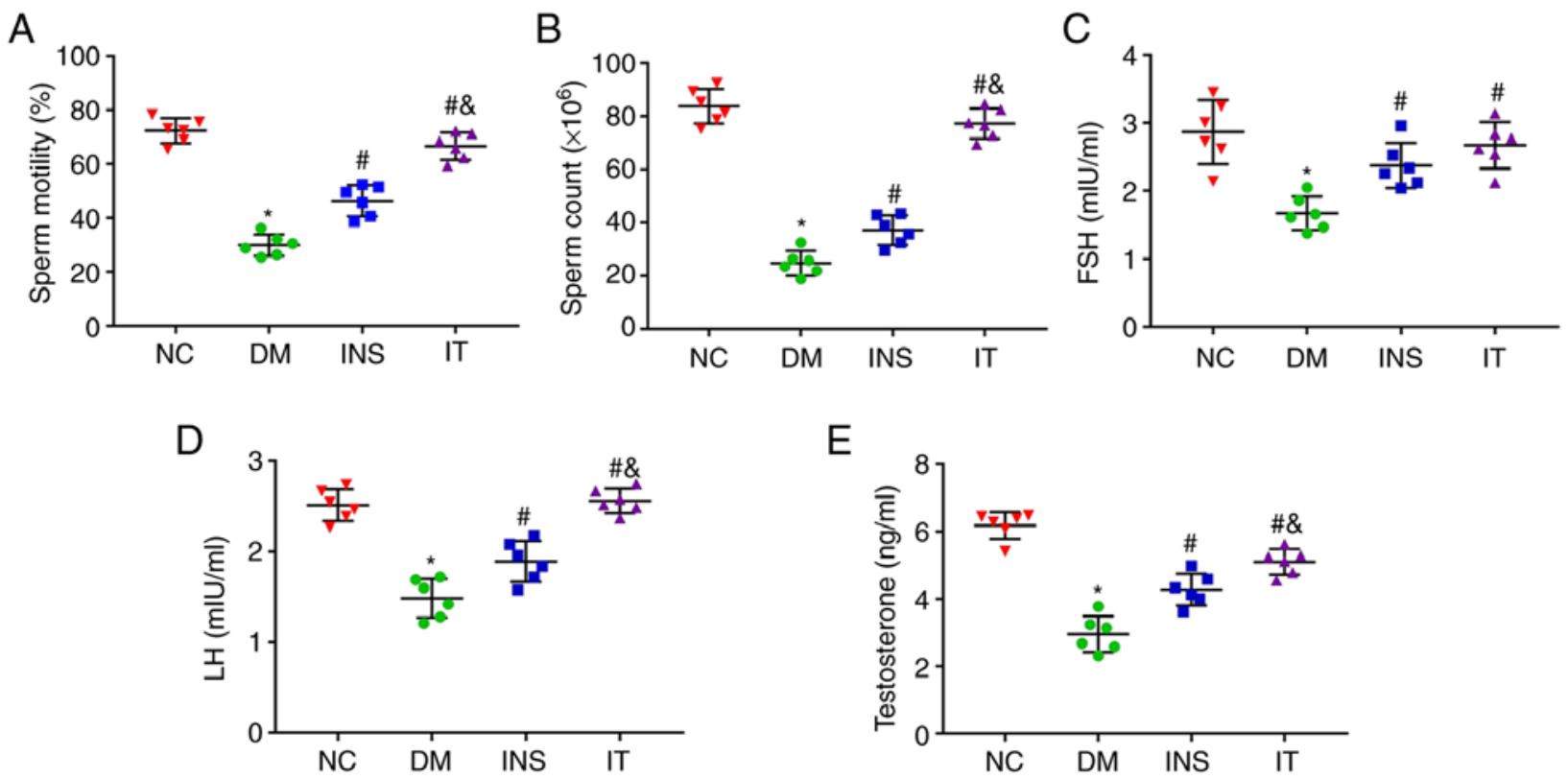

Figure 2. IT improved sperm count and sperm motility and function in diabetic rats. (A and B) Sperm count and sperm motility were measured in each group ( $\mathrm{n}=6$ for each group). The sperm count and the sperm motility were significantly reduced in DM rats. INS and IT treatments increased the sperm count and sperm motility with IT nearly restoring these parameters to normal levels. (C-E) Quantitative detection of (C) FSH, (D) LH and (E) testosterone expression levels in serum, respectively ( $\mathrm{n}=6$ for each group). DM-induced testicular structural damage was accompanied by significant decreases in FSH, LH and testosterone expression levels, which were elevated by INS and IT. Levels of testosterone and LH increased more with IT treatment. "P<0.05 vs. NC; ${ }^{~} \mathrm{P}<0.05$ vs. DM; ${ }^{\circledR} \mathrm{P}<0.05$ vs. INS. FSH, follicle-stimulating hormone; LH, luteinizing hormone; NC, normal control; DM, diabetes mellitus; INS, insulin treatment; IT, islet transplantation.

$10 \mathrm{mg} / \mathrm{ml}$ proteinase $\mathrm{K}$ for antigen retrieval at $37^{\circ} \mathrm{C}$ for $30 \mathrm{~min}$. Endogenous peroxidase was inhibited using $10 \%$ hydrogen peroxide-methanol solution for $10 \mathrm{~min}$. Subsequently, the testis sections were incubated in $1 \%$ Triton X-100 for permeabilizing the cell membrane at room temperature for $20 \mathrm{~min}$. Finally, the sections were blocked with fluorescein-labelled dUTP and TDT-enzyme in proportion for $2 \mathrm{~h}$ at $37^{\circ} \mathrm{C}$ and then incubated with converter-POD peroxidase (HRP labelled fluorescein antibody) for $30 \mathrm{~min}$ at $37^{\circ} \mathrm{C}$, after $30 \mathrm{~min}$ of blockage with $10 \%$ goat serum (OriGene Technologies, Inc.) at room temperature. The sections were washed three times with PBS for 5 min each step. TUNEL-positive cells were counted under the Nikon fluorescence microscope (Eight fields were randomly selected to count the number of positive cells on each slide and the average value of them was calculated; original magnification $\mathrm{x} 400$ ) to compare the degree of apoptosis between different experimental groups.

Histological and immunohistochemical examinations. Testicular tissues were fixed using $4 \%$ formalin for 3 days at $4^{\circ} \mathrm{C}$. After gradient dehydration in ethanol solution and transparency in xylene for $30 \mathrm{~min}$, the tissues were embedded in paraffin and sliced to $5 \mu \mathrm{m}$ thickness. The slides were incubated in an oven at $65^{\circ} \mathrm{C}$ overnight and then rehydrated in a graded ethanol series after $30 \mathrm{~min}$ of deparaffinization in xylene for histological examination. The slides were treated with H\&E (Beijing Solarbio Science \& Technology Co., Ltd.; Hematoxylin for $10 \mathrm{~min}$; and Eosin for $15 \mathrm{sec}$ at room temperature). To detect the ratio of testicular stroma collagen, testicular tissue slides were stained with Masson's trichrome stain according to manufacturers' protocols (Beijing Solarbio Science \& Technology Co., Ltd.). For immunohistochemical staining, the testis sections $(5 \mu \mathrm{m})$ were incubated with $3 \%$ $\mathrm{H} 2 \mathrm{O} 2$ at $37^{\circ} \mathrm{C}$ for $10 \mathrm{~min}$ to inhibit endogenous peroxidase activity. Subsequently, the sections were blocked with $5 \%$ normal goat serum (OriGene Technologies, Inc.) for $30 \mathrm{~min}$. After washing, the samples were incubated with primary antibodies TGF- $\beta 1$ (1:100; Abcam; ab215715; Rabbit monoclonal), CTGF (1:200; Abcam; ab227180; Rabbit polyclonal) and insulin (1:1,0000; Abcam; ab181547; Rabbit monoclonal) overnight at $4^{\circ} \mathrm{C}$. The slices were then incubated with secondary antibody (1:200; cat. no. A0277; Beyotime Institute of Biotechnology; Goat anti-rabbit IgG-HRP), visualized with diaminobenzidine (brown color; OriGene Technologies, Inc.) and analyzed with Image-Pro Plus 6.0 software (Media Cybernetics, Inc.).

Statistical analysis. All data were presented as mean \pm standard deviation. Statistical significance was determined using one-way ANOVA for comparison of $\geq 3$ experimental conditions and Tukey's test was used as a post hoc test following ANOVA. All analyses were performed using SPSS v19.0 (IBM Corp.). $\mathrm{P}<0.05$ was considered to indicate a statistically significant difference.

\section{Results}

Assessment of purity, activity and function of isolated islets and blood glucose levels in diabetic rats following IT. Islet cells were isolated from donor rat pancreas and used for transplantation. The purity of isolated islet cells was evaluated by microscopic observation. Results demonstrated the isolated islet cells had high purity (Fig. 1A). A high level of activity of the isolated islets was also revealed by FDA-PI staining (Fig. 1B). H\&E and immunohistochemical staining was 
A

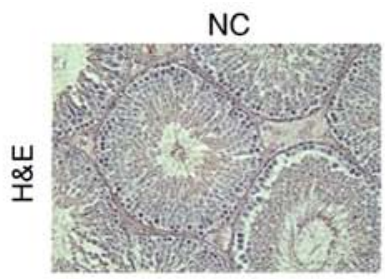

B

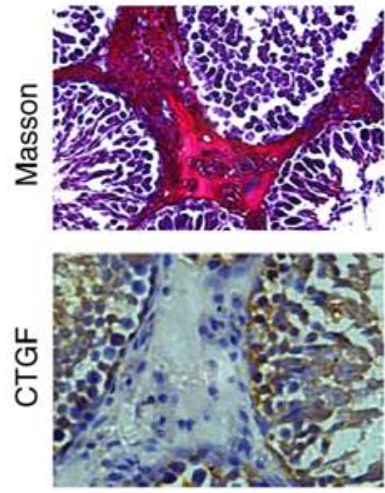

C

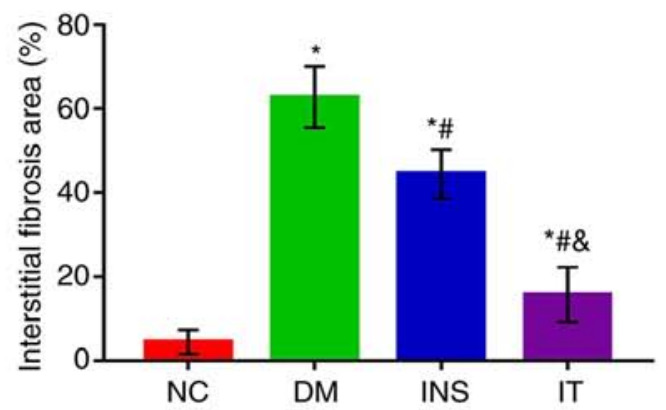

E
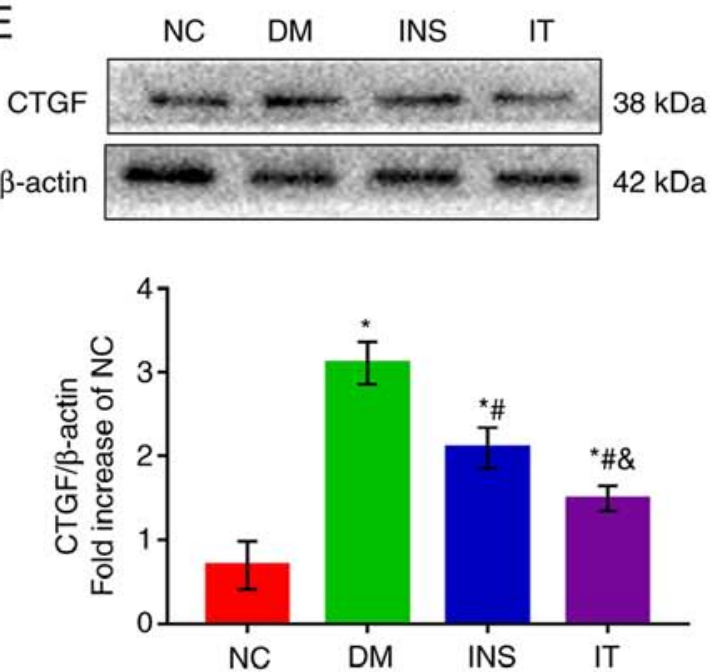

INS
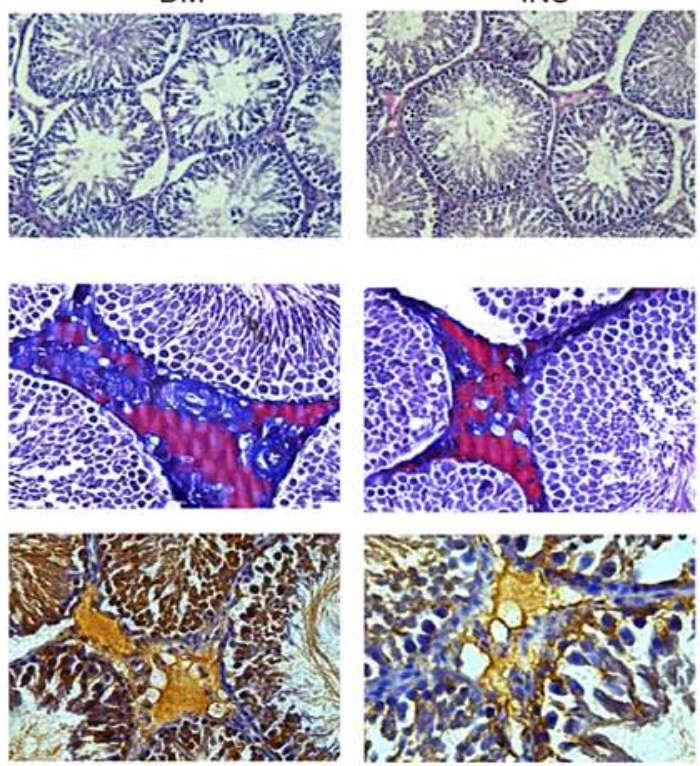

IT
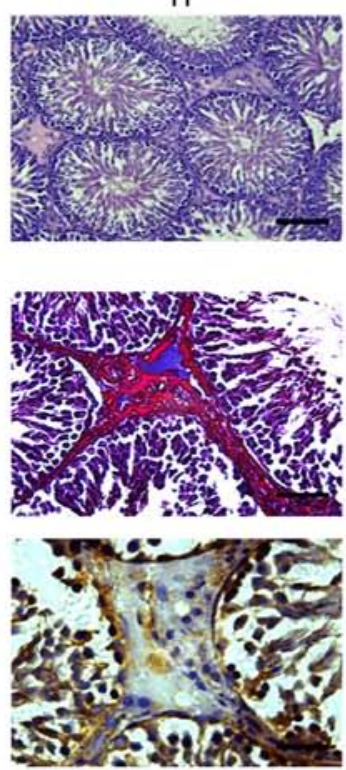

$\mathrm{D}$

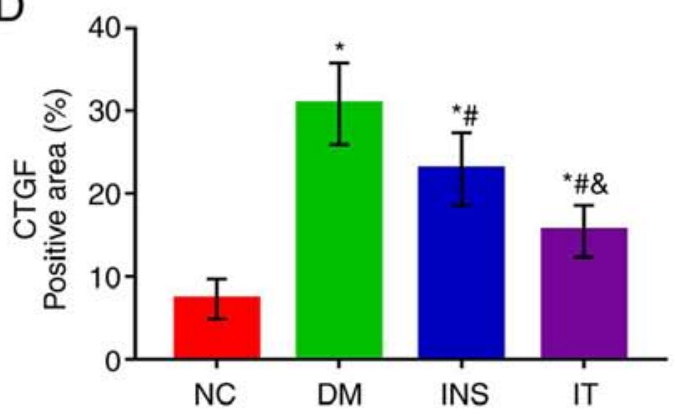

F
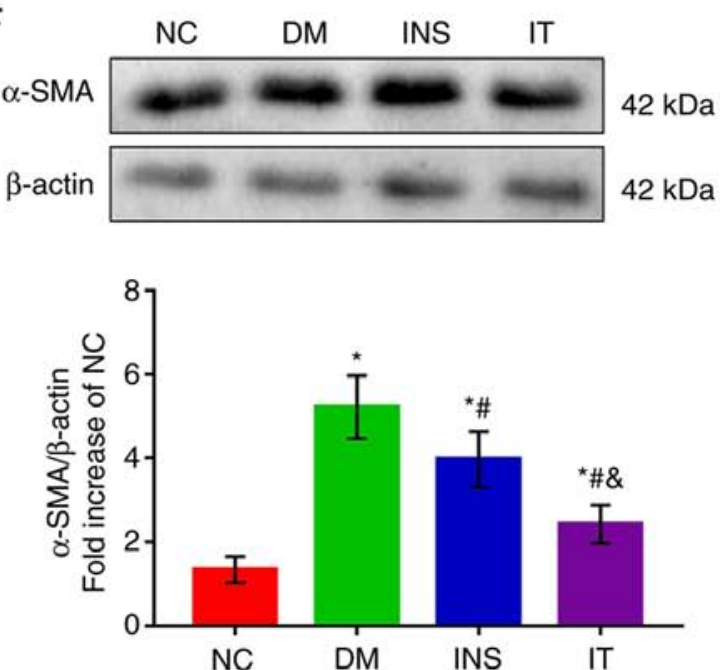

Figure 3. IT attenuated testicular structural damage and reduced DM-induced testicular interstitial fibrosis. (A) hematoxylin and eosin staining of testicular sections in each group $(\mathrm{n}=6$ for each group; scale bar $=25 \mu \mathrm{m}$ ). Testicular sections of the NC group demonstrated normal seminiferous tubules and interstitial structure, including a high number of germ cells, including spermatogonia, spermatocytes and sperm cells. DM group testicular sections demonstrated severe destruction of seminiferous tubules, atrophy of interstitials, decreased Leydig cells and severely reduced germ cells. The INS and IT groups demonstrated recovery and improvement of testicular structure. The IT group had a marked improvement. (B) Masson trichrome staining and CTGF immunohistochemical staining in testicular stroma ( $\mathrm{n}=6$ for each group; scale bar $=25 \mu \mathrm{m})$. (C) Quantitative analysis of the fibrotic area as reflected by Masson's trichrome staining in the testicular stroma. The proportion of collagen in the testicular stroma of DM group was significantly upregulated and IT played a significant protective effect against testicular interstitial fibrosis than INS. (D) Quantifications of CTGF-positive area in the testicular stroma. The positive area of CTGF was larger in the testicular stroma of the DM group but was significantly reduced in the INS group. IT lowered the positive area of CTGF more than INS. (E) Representative western blotting images and quantitative analysis of CTGF protein expression in testis tissues. DM-induced protein expression of CTGF was markedly increased. However, IT significantly attenuated the expression of CTGF compared with INS. (F) Protein expression and quantitative analysis of $\alpha$-SMA in testis tissues. $\alpha$-SMA expression significantly increased in the DM group. INS reduced the $\alpha$-SMA levels with IT showing the lowest levels. "P<0.05 vs. NC. ${ }^{\sharp} \mathrm{P}<0.05$ vs. DM. ${ }^{\&} \mathrm{P}<0.05$ vs. INS. NC, normal control; DM, diabetes mellitus; INS, insulin treatment; IT, islet transplantation; CTGF, connective tissue growth factor; $\alpha$-SMA, $\alpha$-smooth muscle actin. 

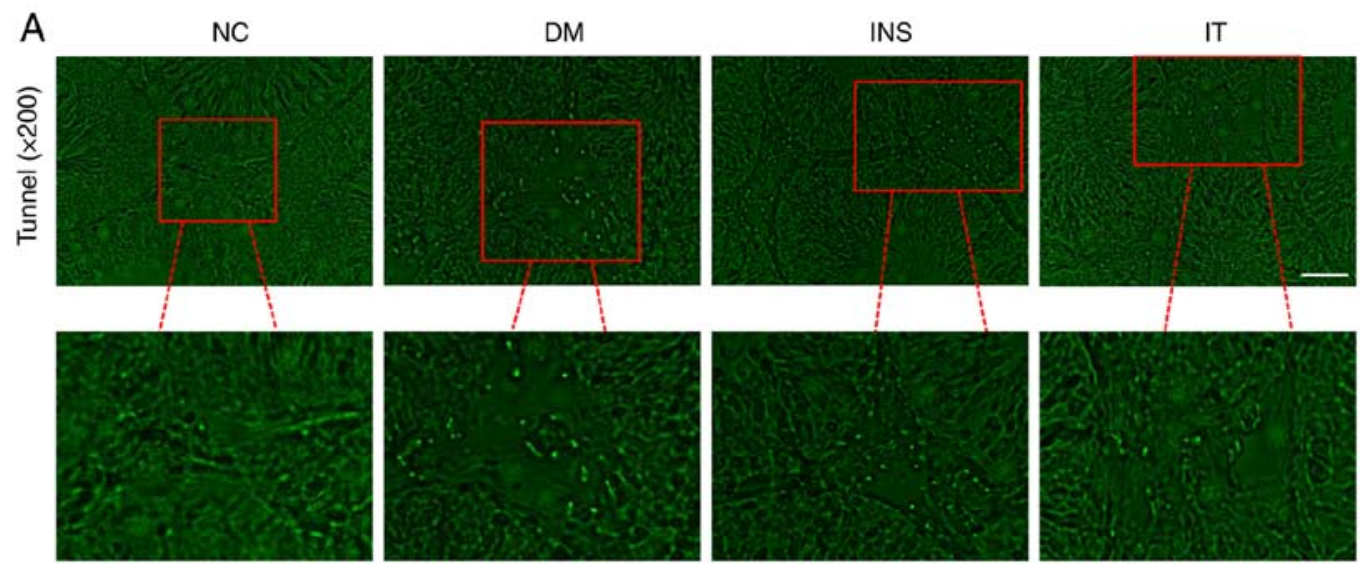

B
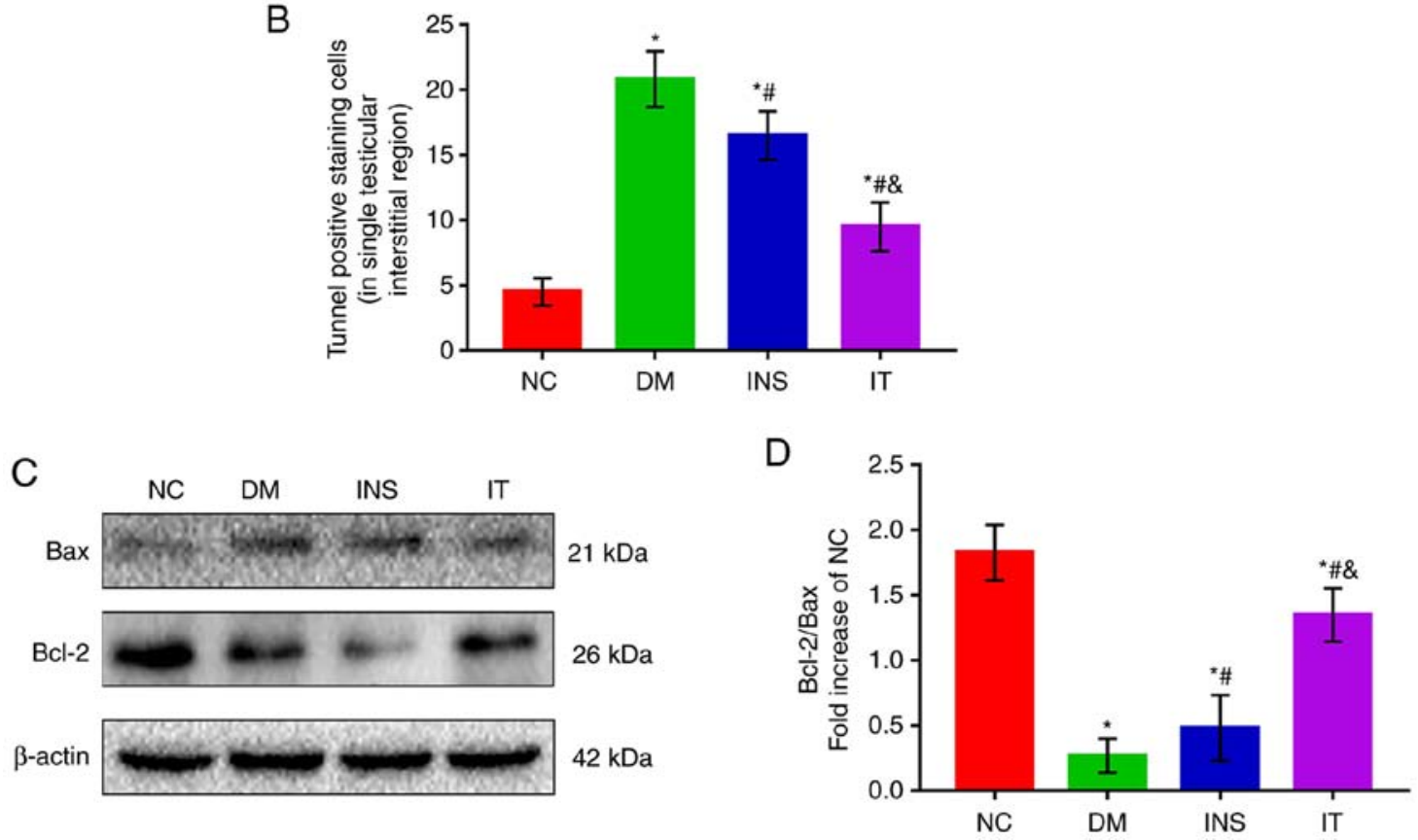

Figure 4. IT attenuated testicular interstitial Leydig cells apoptosis in diabetic rats. (A and B) TUNEL staining was used to demonstrate Leydig cell apoptosis in the testicular stroma $(\mathrm{n}=6$ for each group; scale bar $=25 \mu \mathrm{m})$. DM markedly increased the number of TUNEL-positive cells in the testicular stroma. INS and IT reversed this increase, with IT having more notable effects. (C) Western blot detection and (D) quantification of Bcl-2 and Bax, respectively (n=6 for each). $\mathrm{DM}$ significantly reduced the ratio of $\mathrm{Bcl}-2 / \mathrm{Bax}$. This ratio was increased by INS and IT, with IT showing a significantly higher ratio. ${ }^{*} \mathrm{P}<0.05$ vs. NC; ${ }^{\#} \mathrm{P}<0.05$ vs. DM; ${ }^{\circledR} \mathrm{P}<0.05$ vs. INS. NC, normal control; DM, diabetes mellitus; INS, insulin treatment; IT, islet transplantation.

performed four weeks after transplantation and demonstrated that the islets were well colonized under the renal capsule and exhibited a stable insulin-secreting function (Fig. 1C). Blood glucose levels of the rats in all groups were monitored (Fig. 1D). Diabetic rats treated with insulin or IT demonstrated a significant decrease in blood glucose levels. However, rats in the INS group demonstrated a considerable fluctuation in blood glucose levels compared with the IT group. The blood glucose levels in the IT group were consistently stable in the normal ranges, suggesting that IT was improved in lowering and stabilizing blood glucose levels compared with INS.

IT increased sperm count and motility as well as testosterone, FSH and LH levels in DM rats. Sperms in the epididymis were isolated and collected for counting and determination of motility. The sperm count and motility in the DM group were noted to be lower compared with those in the NC group. However, IT and INS treatment significantly improved the sperm count and motility with IT showing improved results (Fig. 2A and B). To show the effect of IT on the hypothalamicpituitary-gonadal axis, $\mathrm{FSH}, \mathrm{LH}$ and testosterone levels in the serum were measured (Fig. 2C-E). FSH, LH and testosterone levels were significantly reduced in the DM group. However, IT and INS significantly increased the levels of these hormones, with IT showing greater improvement. No significant differences were observed in the hormone levels between the IT and NC groups.

IT treatment alleviated pathological lesions in diabetic rat testes. H\&E staining demonstrated abatement of Leydig cells, disruption of seminiferous tubules and fewer intraluminal spermatozoa in the DM group. INS was able to reverse the structural abnormalities in the testes. However, IT treatment had more favorable results than INS (Fig. 3A). These results suggest that IT treatment is more effective in improving the histological architecture of the testis than INS. 
A

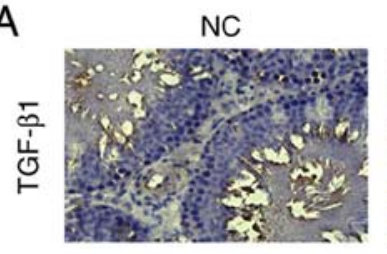

DM

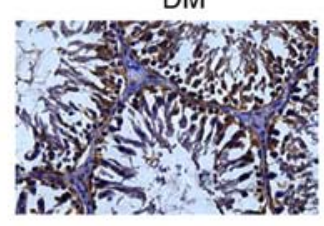

INS



IT

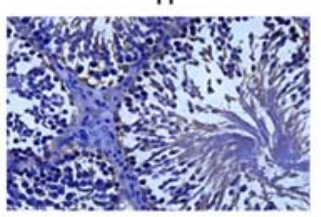

B

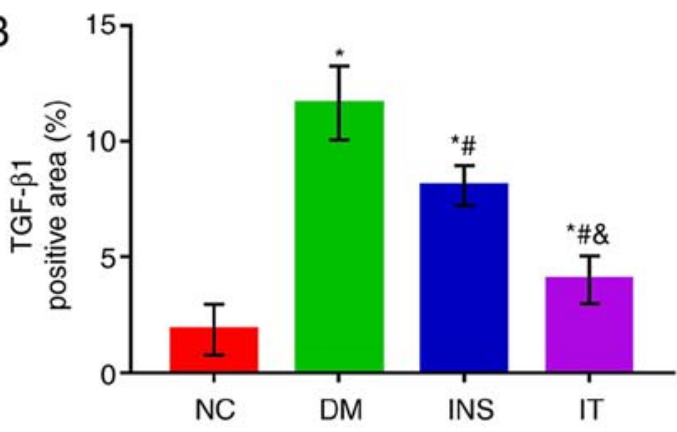

C
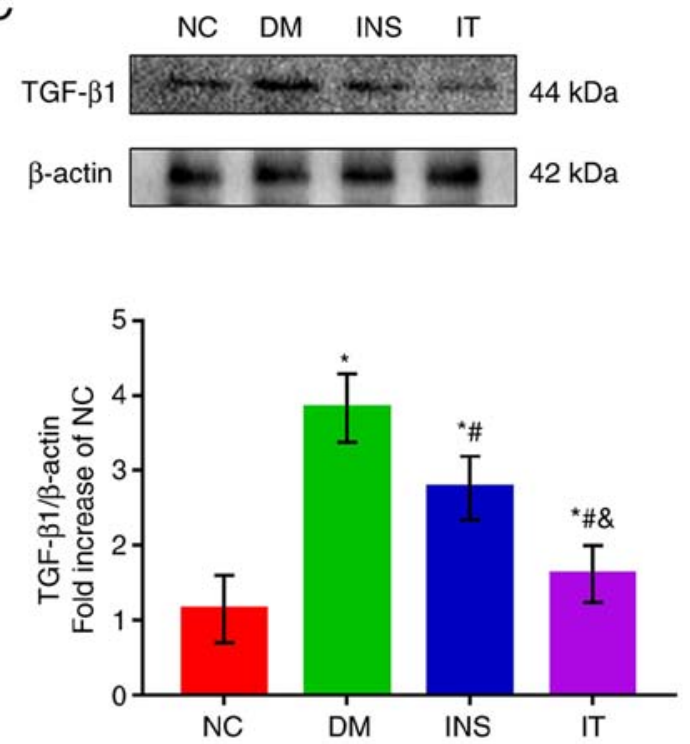

D

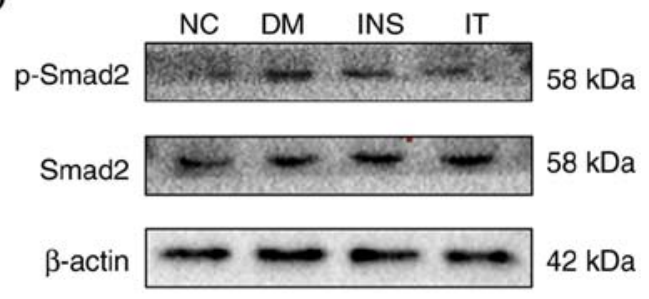

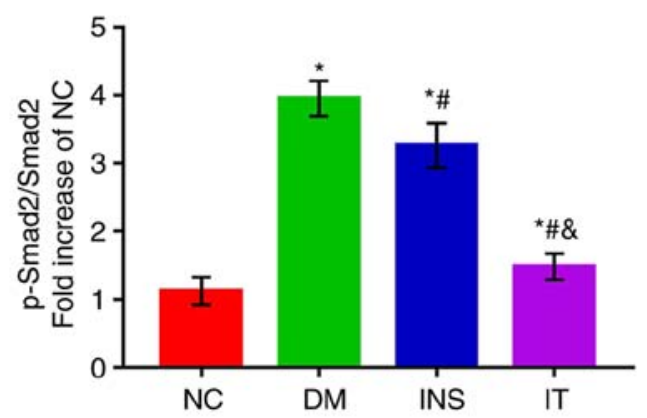

Figure 5. IT downregulated the activation of the TGF- $\beta 1 / \mathrm{Smad} 2$ signaling pathway in the testis tissues of DM rats. (A) Immunohistochemical staining of TGF- $\beta 1$ and (B) quantitative analysis of positive regions in testicular sections, respectively ( $\mathrm{n}=6$ for each group; scale bar $=25 \mu \mathrm{m}$ ). The positive region of TGF- $\beta 1$ was largest in the NC group. INS and IT reduced the positive region of TGF- $\beta 1$, with IT showing the highest reduction. (C) Representative western blot images of TGF- $\beta 1$ and quantifications of its expression levels, respectively. DM induced a marked increase in TGF- $\beta 1$ protein expression in rat testicular tissue. IT group significantly alleviated TGF- $\beta 1$ expression compared with INS. (D) Representative western blot images of p-smad2, Smad 2 and quantitative analysis of their expression levels, respectively. p-Smad2 was significantly elevated in the DM group, whereas INS reduced the expression level of Smad2. IT demonstrated a stronger capability to reduce p-Smad 2 compared with INS. No change was observed in the expression of total Smad 2 across the groups. " $\mathrm{P}<0.05$ vs. NC; " $\mathrm{P}<0.05$ vs. DM; ${ }^{\circledR} \mathrm{P}<0.05$ vs. INS. IT, islet transplantation; DM, diabetes mellitus; NC, normal control; INS, insulin treatment; $\mathrm{p}-$, phosphorylated.

IT inhibited DM-induced testicular interstitial fibrosis. Masson staining demonstrated that the ratio of collagen in the testicular interstitium significantly increased in untreated diabetic rats compared with the NC group. INS also markedly increased the collagen as compared with the DM group that was left untreated. However, IT was more effective in reducing testicular interstitial collagen deposition than INS (Fig. 3B and C). In addition, immunohistochemical staining and western blot analysis demonstrated that DM induced high expression of CTGF in the testicular stroma (Fig. 3B-E) and these results revealed the abnormal deposition of ECM in the testicular stroma. INS significantly attenuated the increase in CTGF with IT producing a marked reduction in CTGF levels. Additionally, western blot analysis demonstrated the abnormally elevated $\alpha$-SMA levels in the DM group. IT was more effective than INS in reducing $\alpha$-SMA levels (Fig. $3 \mathrm{~F}$ ), suggesting that IT could significantly inhibit the differentiation of fibroblasts in the testicular stroma.

IT treatment alleviated DM-induced Leydig cells apoptosis in rat testes. Testicular interstitial fibrosis can lead to apoptosis of Leydig cells, which is closely related to pathological progression of testicular structure and function changes caused by DM (30). Therefore, TUNEL staining was used to detect cell apoptosis (Fig. 4A and B). Apoptosis was observed to be mainly localized to the Leydig cells of testicular 
stroma. A significant increase was observed in the number of TUNEL-positive cells in the DM group compared with the other groups. Although the number of TUNEL-positive cells in the treatment groups was higher compared with the NC group, a significant difference was noted between the IT and INS groups. To confirm the results, the expression levels of Bax and Bcl-2 in the testis of diabetic rats was determined (Fig. 4C and D). The results demonstrated that the Bcl-2/Bax ratio was significantly reduced in the DM group compared with the NC group, whereas in the INS group the ratio was increased compared with the DM group. IT further attenuated the abnormal expression levels of Bcl-2 and Bax.

IT treatment inhibited the DM-induced activation of the TGF- $\beta 1 /$ Smad2 signaling pathway. The TGF- $\beta 1 / \mathrm{Smad} 2$ signaling pathway is highly correlated with testicular function and testicular fibrosis $(31,32)$. Therefore, the effects of IT on the modulation of TGF- $\beta 1$ and Smad 2 expression levels in rat testis tissues were evaluated. The expression levels of TGF- $\beta 1$ and p-Smad 2 markedly decreased after IT or INS treatment as compared with the none treated DM group (Fig. 5A-D). Treatment with IT demonstrated more favorable results than INS.

\section{Discussion}

The present study provided a novel insight into the molecular mechanisms of islet transplantation in improving testicular dysfunction in diabetic rats. The results demonstrated that islet transplantation was superior to insulin therapy in improving blood glucose levels in diabetic rats, which may be attributed to the accurate and real-time insulin secretion of transplanted islets. The present study also illustrated that islet transplantation effectively reversed diabetic-induced testicular interstitial fibrosis, Leydig cells apoptosis, testosterone deficiency and sperm motility. IT demonstrated improved reversal results compared with insulin therapy. These findings suggested that islet transplantation is a reliable clinical cure for DM and offers hope in male diabetic patients with testicular dysfunction especially those with refractory testicular interstitial fibrosis. The present study also demonstrated that these protective properties were associated with the inhibition of the TGF- $\beta 1 / \mathrm{Smad} 2$ signaling pathway.

A number of studies have demonstrated that DM-induced testicular dysfunction is closely interrelated to oxidative stress, inflammatory reaction, apoptosis, angiopathy and other factors $(12,33)$. However, the pathophysiological mechanism remains to be elucidated. Changes in testicular function and structure caused by oxidative stress and inflammation can be easily reversed by effective exogenous antioxidants. However, testicular interstitial fibrosis, caused by long-term high glucose stimulation is difficult to alleviate or reverse (34). Testicular fibrosis impairs the normal structure of the testis and is characterized by the reduction and hardening of the testis (35). Testicular interstitial fibrosis also induces apoptosis of Leydig cells thus impairs secretion of testosterone necessary for maintenance of the number and activity of germ cells (36). Therefore, it is particularly important to study DM-induced testicular interstitial fibrosis. There have been no effective methods for treating or preventing testicular fibrosis and previous research has not fully focused on testicular interstitial fibrosis.

Tight glycemic control by IT has been demonstrated to reverse testicular structural injury through anti-inflammatory and anti-oxidative stress in a diabetic rat model by previous studies (18). However, restoration of the testicular structure does not equate to the restoration of testicular function. Testicular interstitial fibrosis is closely related to decreased testosterone production and poor sperm motility (37). Damaged testicular interstitium also decreases spermatogenic function (38). The present study revealed that IT was effective in improving the testicular interstitial fibrosis in diabetic rats, which manifested as decreased synthesis of ECM and reduced expression of $\alpha$-SMA in the testicular interstitium. IT also effectively inhibited apoptosis of Leydig cells caused by interstitial fibrosis, thereby restoring the secretion of testosterone and normal spermatogenic ability of testis.

In clinical settings, insulin is a common strategy in the control of blood glucose. Previous studies have demonstrated that insulin prevents the development of diabetic complications in the early stages but does not improve or reverse complications in the advanced or late stages $(39,40)$. Previous studies have demonstrated that blood glucose levels of STZ-induced DM in rats fluctuate considerably with insulin therapy $(41,42)$. In addition, insulin in advanced-stage diabetic rats does not reverse the myocardial fibrosis process (28). Consistent with previous studies, the present study revealed that testicular interstitial fibrosis and apoptosis of Leydig cells in the INS group were significantly higher compared with those in the NC and IT groups. The superior effects of IT in retarding diabetic-induced testicular interstitial fibrosis and impairment of spermatogenesis may be attributed to the restoration of $\beta$ cell function and improved blood glucose regulation.

Tissue fibrosis is considered a reparative process in response to cell loss or direct hyperglycemic insult. Excessive fibrosis is inversely correlated with low testosterone and reduced sperm production $(35,43)$. The TGF- $\beta 1 / \mathrm{Smad} 2$ pathway is a wellestablished molecular mechanism in testicular fibrosis (22). The present study demonstrated that DM had a significant effect on the activation of the TGF- $\beta 1 / \mathrm{Smad} 2$ pathway in rat testis. The TGF- $\beta 1 / \mathrm{Smad} 2$ pathway induced expression of CTGF and $\alpha$-SMA, which are important mediators in fibroblast activation (44). IT was demonstrated to inhibit the activity of the TGF- $\beta 1 / \mathrm{Smad} 2$ pathway and suppress the expression of CTGF and $\alpha$-SMA in diabetic testis, resulting in lower testicular fibroblasts activation and ECM deposition. In addition, IT exerted a more significant inhibition of this signaling pathway compared with INS.

The present study had some limitations. First, IT was demonstrated to improve testicular fibrosis after twelve weeks of DM induction. Effects of IT need to be investigated in prolonged DM-induced damage. Second, the present study did not evaluate other non-classical islet peptides, such as C-peptide, GLP-1and GIP that are also secreted by islet cells. These hormones play important roles in regulating the secretion of insulin and have great potential in the treatment of diabetic complications (45). Therefore, additional studies are required to determine the effect of IT via these peptides in improving fibrosis in testis tissues of diabetic rats. 
The present study provided novel insights into the molecular mechanisms underlying the protective effects of IT in the testis of diabetic rats. IT can reverse the apoptosis of Leydig cells and restore testosterone production in advancedstage diabetic rats. Additionally, IT can inhibit testicular interstitial fibrosis associated with the downregulation of the TGF- $\beta 1 /$ Smad 2 pathway. IT is now more widely used in clinics and may replace insulin as the primary method of managing DM (46). Islet transplantation demonstrated a superior role in insulin therapy in improving testicular interstitial fibrosis and restoring testicular spermatogenesis. These results provide a theoretical basis in the treatment of testicular interstitial fibrosis in the advanced stage of DM and offer hope to male diabetic patients to restore fertility and improve their quality of life. Promotion and recognition of islet transplantation needs to be further enhanced.

\section{Acknowledgements}

Not applicable.

\section{Funding}

This project was supported by grants from the National Natural Science Foundation of China (grant no. 80216096), Natural Science Foundation of Zhejiang province (grant no. 84119040G) and Research Incubation Project of The First Affiliated Hospital of Wenzhou Medical University (grant no. FHY2019058).

\section{Availability of data and materials}

All data used or analyzed during the present study are available from the corresponding author on reasonable request.

\section{Authors' contributions}

YCZ, YHW, LJK, MSZ, MMW and CYL performed the experiments. HCW and HWW conceived and designed the research. HWW, YLF and YCZ analyzed the data and drafted the manuscript. HWW and HCW assessed and confirmed the authenticity of all the raw data. CYL reviewed and edited the manuscript. All authors read and approved the final version of the manuscript.

\section{Ethics approval and consent to participate}

All animal experiments were performed according to the regulations of the Animal Experimental Ethical Inspection of Laboratory Animal Centre of Wenzhou Medical University (ID no. wydw-2017-0008) and were performed following the established Guide for the Care and Use of Laboratory Animals.

\section{Patient consent for publication}

Not applicable.

\section{Competing interests}

The authors declare that they have no competing interests.

\section{References}

1. Wang L, Gao P, Zhang M, Huang Z, Zhang D, Deng Q, Li Y, Zhao Z, Qin X, Jin D, et al: Prevalence and ethnic pattern of diabetes and prediabetes in China in 2013. JAMA 317: 2515-2523, 2017.

2. Chung WK, Erion K, Florez JC, Hattersley AT, Hivert MF, Lee CG, McCarthy MI, Nolan JJ, Norris JM, Pearson ER, et al: Precision medicine in diabetes: A consensus report from the American Diabetes Association (ADA) and the European Association for the Study of Diabetes (EASD). Diabetes Care 43: 1617-1635, 2020.

3. Tatone C, Di Emidio G, Barbonetti A, Carta G, Luciano AM, Falone $\mathrm{S}$ and Amicarelli F: Sirtuins in gamete biology and reproductive physiology: Emerging roles and therapeutic potential in female and male infertility. Hum Reprod Update 24: 267-289, 2018.

4. Maresch CC, Stute DC, Alves MG, Oliveira PF, de Kretser DM and Linn T: Diabetes-induced hyperglycemia impairs male reproductive function: A systematic review. Hum Reprod Update 24: 86-105, 2018.

5. Nazmy WH, Elbassuoni EA, Ali FF and Rifaai RA: Proinsulin C-peptide as an alternative or combined treatment with insulin for management of testicular dysfunction and fertility impairments in streptozotocin-induced type 1 diabetic male rats. J Cell Physiol 234: 9351-9357, 2019.

6. Glenn DR, McClure N and Lewis SE: The hidden impact of diabetes on male sexual dysfunction and fertility. Hum Fertil (Camb) 6: 174-179, 2003.

7. Ghosh S, Chowdhury S, Das AK and Sil PC: Taurine ameliorates oxidative stress induced inflammation and ER stress mediated testicular damage in STZ-induced diabetic Wistar rats. Food Chem Toxicol 124: 64-80, 2019.

8. Khosravi Z, Sedaghat R, Baluchnejadmojarad T and Roghani M: Diosgenin ameliorates testicular damage in streptozotocindiabetic rats through attenuation of apoptosis, oxidative stress, and inflammation. Int Immunopharmacol 70: 37-46, 2019.

9. Aksglaede L and Juul A: Testicular function and fertility in men with Klinefelter syndrome: A review. Eur J Endocrinol 168: R67-R76, 2013.

10. Shiraishi K, Takihara $\mathrm{H}$ and Naito K: Quantitative analysis of testicular interstitial fibrosis after vasectomy in humans. Aktuelle Urol 34: 262-264, 2003.

11. Eid AH, Gad AM, Fikry EM and Arab HH: Venlafaxine and carvedilol ameliorate testicular impairment and disrupted spermatogenesis in rheumatoid arthritis by targeting AMPK/ERK and PI3K/AKT/mTOR pathways. Toxicol Appl Pharmacol 364: 83-96, 2019.

12. Arab HH, Gad AM, Fikry EM and Eid AH: Ellagic acid attenuates testicular disruption in rheumatoid arthritis via targeting inflammatory signals, oxidative perturbations and apoptosis. Life Sci 239: 117012, 2019.

13. Kolettis PN and Sabanegh ES: Significant medical pathology discovered during a male infertility evaluation. J Urol 166: 178-180, 2001.

14. Shapiro AM, Pokrywczynska M and Ricordi C: Clinical pancreatic islet transplantation. Nat Rev Endocrinol 13: 268-277, 2017.

15. He Y, Zhang M, Wu Y, Jiang H, Fu H, Cai Y, Xu Z, Liu C, Chen B and Yang T: Aberrant activation of Notch-1 signaling inhibits podocyte restoration after islet transplantation in a rat model of diabetic nephropathy. Cell Death Dis 9: 950, 2018.

16. Preguica I, Alves A, Nunes S, Gomes P, Fernandes R, Viana SD and Reis F: Diet-induced rodent models of diabetic peripheral neuropathy, retinopathy and nephropathy. Nutrients 12: 250 , 2020.

17. Fensom B, Harris C, Thompson SE, Al Mehthel M and Thompson DM: Islet cell transplantation improves nerve conduction velocity in type 1 diabetes compared with intensive medical therapy over six years. Diabetes Res Clin Pract 122: 101-105, 2016.

18. Zhu X, Guo F, Tang H, Huang C, Xie G, Huang T, Li Y, Liu C, Wang $\mathrm{H}$ and Chen $\mathrm{B}$ : Islet transplantation attenuating testicular injury in type 1 diabetic rats is associated with suppression of oxidative stress and inflammation via Nrf-2/HO-1 and NF- $\mathrm{KB}$ pathways. J Diabetes Res 2019: 8712492, 2019.

19. Salama N, Tsuji M, Tamura M and Kagawa S: Transforming growth factor (beta1) in testes of aged and diabetic rats: Correlation with testicular function. Arch Androl 47: 217-226, 2001. 
20. Kabel AM: Zinc/alogliptin combination attenuates testicular toxicity induced by doxorubicin in rats: Role of oxidative stress, apoptosis and TGF- $\beta 1 / \mathrm{NF}-\kappa \mathrm{B}$ signaling. Biomed Pharmacother 97: 439-449, 2018.

21. Hu HH, Chen DQ, Wang YN, Feng YL, Cao G, Vaziri ND and Zhao YY: New insights into TGF- $\beta /$ Smad signaling in tissue fibrosis. Chem Biol Interact 292: 76-83, 2018.

22. Tzavlaki K and Moustakas A: TGF- $\beta$ signaling. Biomolecules 10: 487, 2020.

23. ten Dijke P and Hill CS: New insights into TGF-beta-Smad signalling. Trends Biochem Sci 29: 265-273, 2004.

24. Ramazani Y, Knops N, Elmonem MA, Nguyen TQ Arcolino FO, van den Heuvel L, Levtchenko E, Kuypers D and Goldschmeding R: Connective tissue growth factor (CTGF) from basics to clinics. Matrix Biol 68-69: 44-66, 2018.

25. Hinz B, Celetta G, Tomasek JJ, Gabbiani G and Chaponnier C: Alpha-smooth muscle actin expression upregulates fibroblast contractile activity. Mol Biol Cell 12: 2730-2741, 2001.

26. Yuan X, Pan J, Wen L, Gong B, Li J, Gao H, Tan W, Liang S, Zhang $\mathrm{H}$ and Wang X: MiR-144-3p enhances cardiac fibrosis after myocardial infarction by targeting PTEN. Front Cell Dev Biol 7: 249, 2019.

27. National Research Council (US) Committee for the Update of the Guide for the Care and Use of Laboratory Animals: Guide for the Care and Use of Laboratory Animals. 8th edition. The National Academies Press, Washington, DC, 2011.

28. Wang HW, Chen YH, Chen YY, Huang W, Zhu XD, Ni FB, Wu GD, Xu ZQ, Huang ZQ, Chen BC, et al: Islet transplantation attenuates cardiac fibrosis in diabetic rats through inhibition of TGF- $\beta 1 / S m a d 3$ pathway. Am J Transl Res 10: 2445-2456, 2018.

29. Wu Z, Wang H, Ni F, Jiang X, Xu Z, Liu C, Cai Y, Fu H, Luo J, Chen $\mathrm{W}$, et al: Islet transplantation improved penile tissue fibrosis in a rat model of type 1 diabetes. BMC Endocr Disord 18: 49, 2018.

30. Kilarkaje N, Al-Hussaini H and Al-Bader MM: Diabetes-induced DNA damage and apoptosis are associated with poly (ADP ribose) polymerase 1 inhibition in the rat testis. Eur J Pharmaco 737: 29-40, 2014.

31. Kolettis PN: Evaluation of the subfertile man. Am Fam Physician 67: 2165-2172, 2003.

32. Sun T, Xin Z, Jin Z, Wu Y and Gong Y: Effect of TGF-beta/ Smad signaling on sertoli cell and possible mechanism related to complete sertoli cell-only syndrome. Mol Cell Biochem 319: $1-7,2008$.

33. Li M, Liu Z, Zhuan L, Wang T, Guo S, Wang S, Liu J and Ye Z: Effects of apocynin on oxidative stress and expression of apoptosis-related genes in testes of diabetic rats. Mol Med Rep 7: 47-52, 2013

34. Shiraishi K, Takihara $\mathrm{H}$ and Naito K: Influence of interstitial fibrosis on spermatogenesis after vasectomy and vasovasostomy. Contraception 65: 245-249, 2002.
35. Bhanmeechao C, Srisuwatanasagul S and Ponglowhapan S: Age-related changes in interstitial fibrosis and germ cell degeneration of the canine testis. Reprod Domest Anim 53 (Suppl 3): 37-43, 2018

36. Hoffman WH, Kovacs KT, Gala RR, Keel BA, Jarrell TS, Ellegood JO and Burek CL: Macroorchidism and testicular fibrosis associated with autoimmune thyroiditis. J Endocrinol Invest 14: 609-616, 1991.

37. Wang F, Liu W, Jiang Q, Gong M, Chen R, Wu H, Han R, Chen Y and Han D: Lipopolysaccharide-induced testicular dysfunction and epididymitis in mice: A critical role of tumor necrosis factor alpha. Biol Reprod 100: 849-861, 2019.

38. Kangawa A, Otake M, Enya S, Yoshida T and Shibata M: Histological Changes of the Testicular Interstitium during Postnatal Development in Microminipigs. Toxicol Pathol 47: 469-482, 2019.

39. Cellek S, Foxwell NA and Moncada S: Two phases of nitrergic neuropathy in streptozotocin-induced diabetic rats. Diabetes 52 2353-2362, 2003

40. Choi WS, Kwon OS, Cho SY, Paick JS and Kim SW: Effect of chronic administration of PDE5 combined with glycemic control on erectile function in streptozotocin-induced diabetic rats. J Sex Med 12: 600-610, 2015

41. Poradzka A, Wroński J, Jasik M, Karnafel W and Fiedor P: Insulin replacement therapy in patients with type 1 diabetes by isolated pancreatic islet transplantation. Acta Pol Pharm 70: 943-950, 2013.

42. Beltrán del Río M, Georgiev GI, Cercone R, Tiwari M and Rilo HL: Continuous glucose monitoring analysis as predictor of islet yield and insulin requirements in autologous islet transplantation after complete pancreatectomy. J Diabetes Sci Technol 8: 1097-1104, 2014.

43. Oka S, Shiraishi K and Matsuyama H: Effects of human chorionic gonadotropin on testicular interstitial tissues in men with non-obstructive azoospermia. Andrology 5: 232-239, 2017.

44. Lv W, Zhang L, Cheng X, Wang H, Qin W, Zhou X and Tang B: Apelin Inhibits Angiotensin II-Induced Atrial Fibrosis and Atrial Fibrillation via TGF- $\beta 1 / \mathrm{Smad} 2 / \alpha-$ SMA Pathway. Front Physiol 11: 583570,2020

45. Liu F and Kong Y: GLP-1 receptor agonist on cardiovascular complications of diabetes mellitus. Exp Ther Med 19: 2259-2265, 2020.

46. Roep BO: Improving clinical islet transplantation outcomes. Diabetes Care 43: 698-700, 2020.

This work is licensed under a Creative Commons Attribution-NonCommercial-NoDerivatives 4.0 International (CC BY-NC-ND 4.0) License. 